\title{
Cardiac injury in severe head trauma: a review of literature
}

\author{
Ahmed Hasanin ${ }^{*}$, Dina Zakaria' ${ }^{1}$ Ahmed Allam² \\ 'Department of anesthesia, Cairo University, Egypt \\ ${ }^{2}$ Department of neurosurgery, Cairo University, Egypt
}

\section{Article Info}

\section{Article Notes}

Received: October 13, 2016

Accepted: November 26, 2016

\section{${ }^{*}$ Correspondence:}

Dr. Ahmed Hasanin

Lecturer of anesthesia and critical care medicine, Cairo university, $28 \mathrm{Al}$-alrkam street, almeeraaj, almaadi, Cairo, Egypt, Telephone: +201095076954

Email: ahmedmohamedhasanin@gmail.com

(C) 2016 Hasanin A. This article is distributed under the terms of the Creative Commons Attribution 4.0 International License

\section{Keywords:}

Cardiac injury

Traumatic brain injury

Troponin

Echocardiography

\begin{abstract}
Neurogenic stress cardiomyopathy (NSC) is a stress induced cardiomyopathy reported in various neurological disorders. The most widely accepted theory for the mechanism of NSC is the "catecholamine hypothesis". The available evidence suggests the presence of NSC in patients with severe TBI. The presence of cardiac injury could be a poor prognostic finding in patients with TBI. The possible cardiac injury in TBI patients would make the critical care physicians more cautious with hemodynamic management of these patients. Larger studies with more sophisticated asessment would help to confirm the presence of cardiac injury in these patients.
\end{abstract}

\section{Introduction}

Neurogenic stress cardiomyopathy (NSC) is a stress-related cardiomyopathy that was reported in different neurological conditions. NSC was described as a brain-heart interaction; it was fequently reported in subarachnoid hemorrhage $(\mathrm{SAH})^{1,2}$; NSC was also reported in cerebrovascular strokes ${ }^{3,4}$, status epilepticus ${ }^{5}$, brain tumors ${ }^{6}$, central nervous system infections ${ }^{7}$, and even with emotional stress ${ }^{8}$.

The incidence of NSC in patients with traumatic brain injury (TBI) is not clear; NSC was documented in TBI in few case series ${ }^{9-11}$. Four observational studies investigated the incidence of cardiac injury in patients with TBI (table 1); however, the results of these studies were not consistent. Although the four studies reported ECG changes and elevated serum troponin suggesting the presence of cardiac injury, the presence of functional echocardiographic abnormalities was variable among the four studies.

\section{The current evidence for NSC}

The first study to report NSC in patients with TBI was a retrospective study conducted by Hüttemann et $\mathrm{al}^{12}$ on 51 patients with severe TBI. In the aforementioned study, cardiac dysfunction was reported using trans-esophageal echocardiography in $15 \%$ of patients. In another retrospective study, Prathep and colleagues ${ }^{13}$ investigated the presence of cardiac injury among a larger number of patients (139 patients) with variable grades of TBI. Prathep et al reported abnormal echocardiography in $22.3 \%$ and elevated serum troponin in $24 \%$ of patients; the third study was conducted Serri et $\mathrm{al}^{14}$ on 49 patients with severe TBI; Serri et did not report significant cardiac injury among their cohort. The fourth study was conducted by our group ${ }^{15}$ on 50 patients with severe TBI; we reported the presence of cardiac injury among $50 \%$ of our patients. 


\begin{tabular}{|c|c|c|c|c|}
\hline First author, year & Patients & Study type & Evaluation & Main results \\
\hline Hüttemann, 2002 $2^{(12)}$ & 51 patients with severe TBI ( $50 \%$ brain dead) & Retrospective & TEE & LV dysfunction in $8 \%$ of patients \\
\hline Prathe & $\begin{array}{l}150 \text { patients with mild, moderate, and severe } \\
\text { TBI }\end{array}$ & Retrosp & TTE, troponin and BNP & $\begin{array}{l}\text { RWMA in } 16 \% \text { of patients, LV } \\
\text { dysfunction in } 12 \% \text { of patients }\end{array}$ \\
\hline Serri, 2016 & 49 patients with severe TBI & Pros & TTE, E & No LV dysfunction \\
\hline Hasanin, 2016 & 50 patients with severe $\mathrm{TBI}$ & Prospective & TTE, ECG, and troponin & LV dysfunction in $28 \%$ of patients \\
\hline
\end{tabular}

BNP: brain natriuretic peptide, ECG: Electrocardiograph, LV: left ventricular, RWMA: Regional wall motion abnormalities, TBI: Traumatic brain injury, TEE: Trans-esophageal echocardiography, TTE: Transthoracic echocardiography Table 1: Summary for studies reporting cardiac injury in TBI.

Many reasons could explain the higher incidence of cardiac injury in Hüttemann study ${ }^{12}$ and Hasanin et al study ${ }^{15}$ when compared to Prathep etal study ${ }^{13}$; although the later study included more number of patients, only $56 \%$ of these patients had severe TBI; moreover, the retrospective design of the later study would question its ability to find the incidence of NSC because the echocardiographic examination was conducted according to the clinical need and not according to the study design; thus, some patients with possible cardiac injury might be missed.

Although Serri et $\mathrm{al}^{14}$ reported abnormal ECG and troponin elevation in a considerable number of their patients, they did not report any significant echocardiographic abnormalities; thus, their findings differed from Hasanin et al ${ }^{15}$ findings; this diversity could not be explained by any difference in the methodology; both studies included nearly the same number, age, and type of pathology. In our study, we included severely ill patients with mean APACHE II score of 21, Serri et al did not report the APACHE scores of their patients; however, they reported injury severity score of 32 which is a severe grade of injury. The main difference between the two studies is in the timing of echocardiographic examination which is delayed ( 49 hour) in Serri el al patients.

The four main studies reporting cardiac injury in TBI were characterized by a relatively small sample size; thus, we encourage larger studies with more sophisticated cardiac assessment including more echocardiographic details and more biomarkers.

\section{Mechanism of NSC}

The most widely accepted theory for NSC is the "catecholamine hypothesis" which is defined as "catecholamine-mediated direct cardiac injury". Autonomic stimulation caused by direct brain injury is responsible for NSC. Elevated levels of catecholamines were reported in patients with $\mathrm{SAH}^{16,17}$ and in cases of cardiac stunning after emotional stress ${ }^{8}$. A similar troponin elevation was also reported in an animal experimental model of $\mathrm{SAH}^{18}$. In the aforementioned study, there was a positive correlation between cardiac biomarkers (troponin I and CK-MB) and serum catecholamines (adrenaline and noradrenaline) ${ }^{18}$.
The role of elevation of serum catecholamines in the pathogenesis of cardiac injury in CNS conditions is supported by pathologic features of catecholamineinduced injury in myocardial biopsy specimens taken from brain-dead patients who were donors for solid organ transplantation ${ }^{19}$, and the absence of features of myocardial necrosis in the postmortem specimens taken from patients with SAH who received Propranolol and phentolamine ${ }^{20}$. This role is also confirmed by a study reporting normal coronary angiogram in patients with SAH and elevated ST segment with reversible RWMA ${ }^{21}$.

The most widely used biomarker in diagnosis of NSC is serum troponin I. Serum troponin elevation was a clue marker in diagnosis in NSC in patients with $\mathrm{TBI}^{13-15}$, $\mathrm{CVS}^{3,4}$, and $\mathrm{SAH}^{16,22}$. Serum toponin showed a senstivity of $100 \%$ and specificity of $91 \%$ in prediction of cardiac injury in patients with SAH. Brain natriuretic peptide (BNP) was another biomarker used by Prathep et $\mathrm{al}^{13}$ in assessment of NSC in patients with TBI. More biomarkers are encouraged for assessment of NCS in different patient populations.

\section{Cardiac injury and patient outcomes}

The relation between cardiac injury and outcome was reported in different CNS conditions such as SAH, CVS, and TBI. The severity of cardiac injury was a risk factor of mortality in many reports. In patients with $\mathrm{SAH}$, there was an association between ECG abnormalities and outcomes ${ }^{23,24}$; there was also an association between left ventricular dysfunction and mortality ${ }^{25}$. In a large retrospective study on 4695 patients with SAH, Zarof et $\mathrm{al}^{26}$ reported an increased mortality and stroke rates in patients with cardiac injury compared to patients without cardiac injury. In patients with cerebrovascular strokes, there was an association with serum troponin and patients' outcome ${ }^{3,4,27}$. In TBI there was an association between cardiac injury and poor outcomes ${ }^{13,15}$; moreover, a neurogenic cardiac injury score (NCIS) was developed by our group to grade the severity of cardiac injury, the score was based upon the presence of one or more of three components: elevated serum troponin, abnormal echocardiogram, and hypotension. We reported that increased NCIS is an independent risk factor for mortality ${ }^{15}$. 


\section{Management and clinical implications of NSC}

Although cardiac protection using propranolol and phentolamine was suggested by Neil-Dwyer et $\mathrm{al}^{20}$, they only reported the absence of histopathological necrosis in the post-mortem specimens of the patients receiving the drugs without any improvement in the outcomes. It was suggested that the severity if cardiac injury is related to the severity of head injury; thus, management of cardiac injury might not improve the patient outcome; however, this suggestion should be confirmed in future trials. The awareness with cardiac injury in patients with TBI would be make the anesthetists and critical care physicians more cautious when dealing with these patients especially with induction of anesthesia as well as sedation in the intensive care units. Risk stratification of TBI patients should not ignore the presence of cardiac injury as an independent risk factor for poor outcomes.

In conclusion, the available collective evidence suggests the presence of cardiac injury in patients with severe TBI. The presence of cardiac injury could be a poor prognostic finding. Larger studies with more sophisticated measures would help to confirm the presence of cardiac injury in these patients an would also investigate its mechanism.

\section{References}

1. Banki N, Kopelnik A, Tung P, et al. Prospective analysis of prevalence distribution and rate of recovery of left ventricular systolic dysfunction in patients with subarachnoid hemorrhage. J Neurosurg. 2006 Jul; 105(1): 15-20.

2. Temes RE, Tessitore E, Schmidt JM, et al. Left ventricular dysfunction and cerebral infarction from vasospasm after subarachnoid hemorrhage. Neurocrit Care. 2010 Dec; 13(3): 359-65.

3. James P, Ellis CJ, Whitlock RM, et al. Relation between troponin T concentration and mortality in patients presenting with an acute stroke observational study. BMJ. 2000; 320(7248): 1502-4

4. Song HS, Back JH, et al. Cardiac troponin T elevation after stroke Relationships between elevated serum troponin $\mathrm{T}$ stroke location and prognosis. J Clin Neurol. 2008; 4(2): 75-83.

5. Stöllberger C, Huber JO, Enzelsberger B, et al. Fatal outcome of epileptic seizure-induced takotsubo syndrome with left ventricular rupture. Eur J Neurol. 2009 Jun; 16(6): e116-7.

6. Koepp M, Kern A, Schmidt D. Electrocardiographic changes in patients with brain tumors. Arch Neurol. 1995 Feb; 52(2): 152-5.

7. Gelow J, Kruer M, Yadav V, et al. Apical ballooning resulting from limbic encephalitis. Am J Med. 2009 Jun; 122(6): 583-6.

8. Wittstein IS, Thiemann DR, Lima JAC, et al. Neurohumoral features of myocardial stunning due to sudden emotional stress. N Engl J Med. 2005 Feb 10; 352(6): 539-48.

9. Riera M, Llompart-Pou JA, Carrillo A, et al. Head injury and inverted Takotsubo cardiomyopathy. J Trauma. 2010 Jan; 68(1): E13-5.

10. Bahloul M, Chaari AN, Kallel H, et al. Neurogenic pulmonary edema due to traumatic brain injury evidence of cardiac dysfunction. Am J Crit Care. 2006 Sep; 15(5): 462-70.
11. Lin $\mathrm{X}, \mathrm{Xu} \mathrm{Z}$, Wang $\mathrm{P}$, et al. Role of PiCCO monitoring for the integrated management of neurogenic pulmonary edema following traumatic brain injury A case report and literature review. Exp Ther Med. 2016 Oct; $12(4):$ 2341-7.

12. Hüttemann E, Schelenz C, Chatzinikolaou K, et al. Left ventricular dysfunction in lethal severe brain injury Impact of transesophageal echocardiography on patient management. Intensive Care Med. 2002; 28(8): 1084-8.

13. Prathep S, Sharma D, Hallman M, et al. Preliminary report on cardiac dysfunction after isolated traumatic brain injury. Crit Care Med. 2014; 42(1): 142-7.

14. Serri K, El Rayes M, Giraldeau G, et al. Traumatic brain injury is not associated with significant myocardial dysfunction an observational pilot study. Scand J Trauma Resusc Emerg Med. 2016 Jan; 24(1): 31.

15. Hasanin A, Kamal A, Amin S, et al. Incidence and outcome of cardiac injury in patients with severe head trauma. Scand J Trauma Resusc Emerg Med. 2016; 24: 58.

16. Parekh N, Venkatesh B, Cross D, et al. Cardiac troponin I predicts myocardial dysfunction in aneurysmal subarachnoid hemorrhage. J Am Coll Cardiol. 2000 0ct; 36(4): 1328-35.

17. Naredi S, Lambert G, Edén E, et al. Increased sympathetic nervous activity in patients with nontraumatic subarachnoid hemorrhage. Stroke. 2000; 31(4): 901-6.

18. Masuda T, Sato K, Yamamoto SI, et al. Sympathetic nervous activity and myocardial damage immediately after subarachnoid hemorrhage in a unique animal model. Stroke. 2002; 33(6): 1671-6.

19. Berman M, Ali A, Ashley E, et al. Is stress cardiomyopathy the underlying cause of ventricular dysfunction associated with brain death. J Heart Lung Transplant. 2010 Sep; 29(9): 957-65.

20. Neil-Dwyer G, Walter P, Cruickshank JM, et al. Effect of propranolol and phentolamine on myocardial necrosis after subarachnoid haemorrhage. Br Med J. 1978 Oct 7; 2(6143): 990-2.

21. Kuroiwa T, Morita $\mathrm{H}$, Tanabe $\mathrm{H}$, et al. Significance of ST segment elevation in electrocardiograms in patients with ruptured cerebral aneurysms. Acta Neurochir (Wien). 1995 Jan; 133(3-4): 141-6.

22. Horowitz MB, Willet D, Keffer J. The use of cardiac troponin-I (cTnI) to determine the incidence of myocardial ischemia and injury in patients with aneurysmal and presumed aneurysmal subarachnoid hemorrhage. Acta Neurochir (Wien). 1998 Jan; 140(1): 87-93.

23. Coghlan LA, Hindman BJ, Bayman EO, et al. Independent associations between electrocardiographic abnormalities and outcomes in patients with aneurysmal subarachnoid hemorrhage findings from the intraoperative hypothermia aneurysm surgery trial. Stroke. 2009 Feb; 40(2): 412-8.

24. Ichinomiya T, Terao Y, Miura K, et al. QTc interval and neurological outcomes in aneurysmal subarachnoid hemorrhage. Neurocrit Care. 2010 Dec; 13(3): 347-54.

25. Sugimoto K, Watanabe E, Yamada A, et al. Prognostic implications of left ventricular wall motion abnormalities associated with subarachnoid hemorrhage. Int Heart J. 2008 Jan; 49(1): 75-85.

26. Zaroff JG, Leong J, Kim H, et al. Cardiovascular predictors of long-term outcomes after non-traumatic subarachnoid hemorrhage. Neurocrit Care. 2012 Dec; 17(3): 374-81.

27. Di Angelantonio E, Fiorelli M, Toni D, et al. Prognostic significance of admission levels of troponin I in patients with acute ischaemic stroke. J Neurol Neurosurg Psychiatry. 2005; 76(1): 76-81. 